\title{
Estudo da síndrome da ceratoconjuntivite seca de pacientes soropositivos para o vírus da imunodeficiência adquirida humana tipo 1 e com síndrome da imunodeficiência adquirida, em uso ou não de terapia anti-retroviral combinada(HAART)
}

\author{
Studyofkeratoconjunctivitis sicca in patients infected with thehuman immunodeficiency \\ virus type 1 and acquired immune deficiency syndrome, in use or not of highly active anti- \\ retroviral therapy $(H A A R T)$
}

Márcia Lopes Rodrigues ${ }^{1}$

Maria de Lourdes Veronesi Rodrigues ${ }^{2}$ João Alberto Holanda de Freitas ${ }^{3}$

\section{RESUMO}

Objetivos: Verificar a presença de olho seco e de alterações no epitélio conjuntival em pacientes com sorologia positiva para o HIV-1 e com AIDS; relacionar as eventuais alterações com as características e com outras condições oculares e sistêmicas dos pacientes; estudar a influência da terapia anti-retroviral combinada na ceratoconjuntivite seca (KCS), nos pacientes com AIDS. Métodos: Foram estudados pacientes com confirmação laboratorial da infecção pelo HIV, divididos em 2 grupos: I. HIV+ (sem diagnóstico clínico e com contagem de CD4+ acima de 200 células $/ \mathrm{mm}^{3}$ ) e II. Pacientes com AIDS (contagem de CD4+ abaixo de 200 células e/ou manifestação clínica). Foram estudadas alterações oculares, dados laboratoriais, análise do filme lacrimal e estudo da citologia de impressão conjuntival. Método estatístico: qui-quadrado.Resultados: Incluídos 43 pacientes do grupo Ie 77 do grupo II. Após a introdução do HAART houve queda significativa das manifestações oculares internas; entretanto, o mesmo não ocorreu com as externas. Dos pacientes que apresentaram quadro clínico de olho seco, 65,1\% eram do sexo masculino. As alterações do teste de Schirmer e tempo de ruptura do filme lacrimal não estiveram relacionados com a gravidade da doença pelo HIV e nem com a contagem de CD4+.Todos os pacientes com alterações na citologia de impressão apresentavam KCS e $88,8 \%$ pertenciam ao grupo II. Observou-se aumento da freqüência dessas alterações nos pacientes com tempo de doença superior a 4 anos. Conclusão: $O$ decréscimo da produção lacrimal não esteve relacionado com a gravidade da infecção pelo HIV e a introdução do HAART não interferiu na freqüência da síndrome de olho seco nos pacientes HIV positivos.

Descritores: Ceratoconjuntivite seca; Soropositividade para HIV; Síndrome da imunodeficiência adquirida; Antivirais/uso terapêutico; Terapia combinada; HIV-1/efeito de drogas

Endereço para correspondência: Márcia Lopes Rodrigues, Av. General Osório 1415, Ed. Buriti, apto. 31

Sorocaba (SP) CEP 18060-001

E-mail: musicmed@ig.com.br

Apoio financeiro: CAPES

Recebido para publicação em 16.10.2002

Versão revisada recebida em 21.03.2003

Aprovação em 20.11.2003

Nota Editorial: Pela análise deste trabalho e por sua anuência na divulgação desta nota, agradecemos ao Dr. Haroldo Vieira de Moraes Jr.

\section{INTRODUÇÃO}

A síndrome de imunodeficiência adquirida (AIDS) foi inicialmente reconhecida em julho de 1981 nos Estados Unidos, apesar de atualmente a maior parte dos cientistas admitir que o vírus da imunodeficiência humana (HIV)é 
284 Estudo da síndrome da ceratoconjuntivite seca de pacientes soropositivos para o vírus da imunodeficiência adquirida humana tipo 1 e com síndrome da imunodeficiência adquirida, em uso ou não de terapia anti-retroviral combinada (HAART)

originário de primatas, na África, possivelmente decorrente de transmissão cruzada entre espécies ${ }^{(1-2)}$.

A transmissão inicial de retrovírus de primatas para o homem, pode ter ocorrido de várias maneiras: mordidas, escoriações, ou qualquer contato com seu sangue. Contudo, coincidentemente, a pandemia da AIDS acompanhou a utilização, em larga escala, de primatas em experimentação biológica, inclusive xenotransplantes ${ }^{(3)}$.

Segundo dados da Organização Mundial de Saúde (OMS), estima-se que havia cerca de 40 milhões de pessoas contaminadas pelo vírus no mundo até dezembro de 2001, sendo a característica principal da infecção pelo HIV, a depleção progressiva do subgrupo auxiliar / indutor dos linfócitos T4 (4-6).

A classificação sistemática para definição de um caso de AIDS foi proposta em 1982 pelo Centers for Disease Control (CDC) Atlanta - EUA, sendo revisada em 1997. Atualmente, está em vigência o sistema da classificação baseado na mensuração de linfócitos T CD4 +, já que a queda nos níveis destes linfócitos é uma das principais características da doença ${ }^{(7)}$.

Quanto às alterações oculares na AIDS, anteriormente à introdução da terapia anti-retroviral combinada (HAART), ao nível do segmento anterior, eram freqüentes o sarcoma de Kaposi, o linfoma de Burkitt, a microvasculopatia conjuntival, as conjuntivites não específicas, a ceratoconjuntivite seca, e as iridociclites, entre outras. As principais alterações do segmento posterior eram os exsudatos algodonosos, a retinite por citomegalovírus, por herpes simples e varicela zoster, por HIV, por toxoplasmose, a retinite sifilítica e as endoftalmites infecciosas ${ }^{(8)}$.

Entre as doenças oculares externas, diversos estudos em pacientes com teste anti-HIV positivo, relatam uma importante prevalência de olho seco entre os pacientes com $\operatorname{AIDS}^{(8-14)}$, em período anterior ao HAART.

O epitélio normal da conjuntiva ocular e da córnea, que é estratificado e não queratinizado, na ceratoconjuntivite seca normalmente apresenta diminuição na contagem das células caliciformes e um epitélio não secretório e queratinizado. Esta transição patológica é chamada de metaplasia escamosa. A severidade das alterações da superfície ocular é paralela ao grau de metaplasia escamosa ${ }^{(15)}$. Contudo, a patogênese da diminuição das células caliciformes e da metaplasia escamosa e como se relacionam ao olho seco, ainda permanece obscura ${ }^{(16-17)}$.

Uma boa técnica para o estudo da superfície conjuntival é a análise feita com a escova citológica "Cytobrush small" (Cytobrush-S ${ }^{\circledR}$; Medscand, Malmö, Sweden).Trata-se de uma escova semelhante à escova citológica vaginal, com grande homogeneidade entre as amostras. Outra opção é o milipore; porém a dificuldade de determinar a pressão e tempo necessário de contato com a conjuntiva e o diâmetro dos poros, dificulta a coleta de amostras homogêneas ${ }^{(18-20)}$.

\section{OBJETIVOS}

Verificar a presença de olho seco e de metaplasia escamosa no epitélio conjuntival em pacientes com sorologia positiva para o vírus HIV-1 e com AIDS; relacionar as eventuais al- terações com as características e com outras condições oculares e sistêmicas dos pacientes; e estudar a influência da terapia anti-retroviral combinada na ceratoconjuntivite seca, nos pacientes com AIDS.

\section{MÉTODOS}

Trata-se de um estudo observacional, transversal, quantitativo e analítico, realizado no Conjunto Hospitalar de Sorocaba - CHS, no período de março de 1998 a dezembro de 2000, onde foram incluídos pacientes com idade igual ou superior a 18 anos, de ambos os sexos.

O trabalho foi aceito pelo Comitê de Ética do Hospital e todos os pacientes foram informados sobre as razões pelas quais este estudo estava sendo realizado e aceitaram participar.

Foram colhidos dados de identificação (nome, sexo, idade), características epidemiológicas (modo de contaminação), manifestações clínicas oftalmológicas, dados laboratoriais (anticorpos anti-HIV, dosagem de linfócitos T CD4+ e T $\mathrm{CD} 8+$ ) e terapêuticos (terapia oral anti-retroviral).

Os pacientes foram encaminhados com diagnóstico positivo de infecção pelo vírus HIV-1, independentemente de apresentarem ou não AIDS ou queixas oftalmológicas. A classificação do quadro clínico sistêmico foi feito de acordo com as normas do CDC, sendo os pacientes divididos em assintomáticos, pacientes com infecção sintomática precoce pelo HIV e AIDS propriamente dita.

Foram excluídos desta análise, pacientes que estivessem em uso de algum tipo de medicação tópica ocular, que apresentavam doenças autoimunes (síndrome de Sjögren), tracoma, síndrome de Stevens-Johnson, penfigóide ocular, queimadura química ocular e paralisia facial periférica.

Os pacientes foram divididos em 2 grupos: Grupo I: pacientes HIV+ (sem diagnóstico clínico e laboratorial de AIDS propriamente dita - contagem de linfócitos T CD4+ acima de 200 células $/ \mathrm{mm}^{3}$ ). Grupo II: pacientes com AIDS (manifestação clínica e/ou contagem de linfócitos T CD4+ abaixo de 200 células $/ \mathrm{mm}^{3}$ ), sendo estudados um total de 120 pacientes.

Com relação à síndrome de olho seco, todos os pacientes foram estudados segundo 3 critérios: diagnóstico sintomático de olho seco (sensação de ardência, secura nos olhos, desconforto ocular, queimação, fotofobia, prurido); diagnóstico clínico de olho seco (hiperemia, perda do brilho da conjuntiva bulbar, interrupção do menisco lacrimal, presença de muco no canto dos olhos); e testes objetivos de olho seco (teste de Schirmer I, do teste coloração com rosa bengala, e do tempo de ruptura do filme lacrimal - BUT). Estabeleceu-se como olho seco a presença de pelo menos 3 sintomas e 3 sinais segundo os diagnósticos sintomático e clínico respectivamente, e de algum dos seguintes critérios: teste de Schirmer inferior a $5 \mathrm{~mm}$, testes de rosa bengala II ou III e BUT igual ou inferior a 10 segundos $^{(21)}$.

Para investigação laboratorial da citologia de impressão 
conjuntival adotou-se a escova citológica - Cytobrush-S ${ }^{\circledR}$, sendo a coleta feita a partir da parte superior da conjuntiva bulbar (região nasal) de ambos os olhos. Os esfregaços celulares eram fixados em lâmina com Citofix e corados pelo método hematoxilina-eosina, sendo estudado a presença de metaplasia escamosa do epitélio conjuntival.

Com relação à terapia oral, os pacientes de cada grupo foram divididos em 2 subgrupos: ausência de terapia oral e pacientes em uso de terapia oral combinada.

Análise estatística: teste do Qui-quadrado nível de significância de 0,05 ou $5 \%$.

\section{RESULTADOS}

Foram incluídos no estudo 120 pacientes com sorologia positiva para o vírus HIV-1, sendo 43 pacientes do Grupo I e 77 pacientes do Grupo II. Destes, 61,6\% do sexo masculino e 38,3\% do sexo feminino, sem diferença estatisticamente significativa entre os dois grupos quanto ao sexo e idade $(p>0,05)$.

Quanto à classificação do quadro clínico sistêmico, 23,7 \% dos pacientes eram assintomáticos, 9,2\% tinham infecção sintomática precoce pelo HIV e 67,1\% tinham AIDS.

Com relação ao tratamento oral preconizado 78 pacientes (65\%) estavam em uso de HAART, sendo 19 pacientes do Grupo I e 59 pacientes do Grupo II (Tabela 1).

Dos pacientes estudados, 31 pacientes $(25,8 \%)$ apresentaram diagnóstico clínico sintomático de olho seco, sendo 4 pacientes do Grupo I e 27 pacientes do Grupo II. Com relação ao sexo, prevaleceu o masculino em ambos os grupos: $65,1 \%$ dos pacientes acometidos (Tabela 2).

Quanto aos testes de função lacrimal, 22,5\% do total dos pacientes estudados apresentaram alteração nos testes de Schirmer e tempo de ruptura do filme lacrimal, e $25,8 \%$ no teste de coloração com rosa bengala. Ambos resultados de Schirmer e BUT não estiveram relacionados com a severidade da doença pelo HIV e nem com a contagem de linfócitos T CD4+ $(\mathrm{p}>0,05)$ (Tabela 3$)$.

Com relação à citologia de impressão conjuntival, 18 pacientes apresentaram metaplasia escamosa, sendo 2 do Grupo I e 16 pacientes do Grupo II (Tabela 4). Observou-se que entre os pacientes com metaplasia escamosa, 32,2\% apresentavam AIDS há mais de 4 anos.

Dos pacientes com e sem AIDS que faziam uso de HAART, $21,7 \%$ dos pacientes apresentaram ceratoconjuntivite seca e $16,6 \%$ dos pacientes apresentaram metaplasia escamosa. Entre os pacientes que não estavam em uso de HAART, $11 \%$ apresentaram metaplasia escamosa e $33,3 \%$ apresentaram ceratoconjuntivite seca.

\section{DISCUSSÃO}

As doenças associadas a AIDS apresentaram uma queda significativa no que diz respeito às manifestações oculares

\begin{tabular}{|c|c|c|}
\hline $\begin{array}{l}\text { Grupos } \\
\text { Conduta terapêutica }\end{array}$ & $\begin{array}{l}\text { Grupo I } \\
\text { N pac. }^{\circ}\end{array}$ & $\begin{array}{l}\text { Grupo II } \\
N^{\circ} \text { pac. }\end{array}$ \\
\hline Ausência de HAART & 24 & 18 \\
\hline HAART & 19 & 59 \\
\hline \multirow[t]{2}{*}{ Total } & 43 & 77 \\
\hline & & $p>0,05$ \\
\hline \multicolumn{3}{|c|}{ 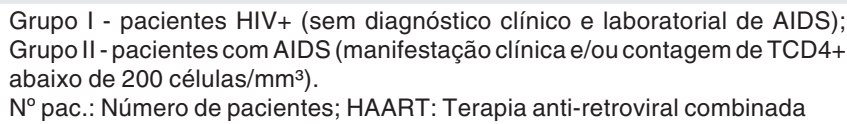 } \\
\hline
\end{tabular}

Tabela 2. Distribuição dos pacientes com diagnóstico clínico sintomático de olho seco, segundo a conduta terapêutica adotada

$\begin{array}{lcc}\begin{array}{r}\text { Grupos } \\ \text { Conduta terapêtica }\end{array} & \begin{array}{c}\text { Grupo I } \\ \mathbf{N}^{\circ} \text { pac. }\end{array} & \begin{array}{c}\text { Grupo II } \\ \mathbf{N}^{\circ} \text { pac. }\end{array} \\ \text { Ausência de HAART } & 2 & 12 \\ \text { HAART } & 2 & 15 \\ \text { Total } & 4 & 27 \\ & & \mathrm{p}>0,05\end{array}$

Grupo I - pacientes HIV+ (sem diagnóstico clínico e laboratorial de AIDS); Grupo II - pacientes com AIDS (manifestação clínica e/ou contagem de TCD4+ abaixo de 200 células $/ \mathrm{mm}^{3}$ ).

No pac.: Número de pacientes; HAART: Terapia anti-retroviral combinada

Tabela 3. Distribuição dos pacientes que apresentaram diagnóstico de ceratoconjuntivite seca, segundo os testes objetivos de olho seco

$\begin{array}{lcc}\quad \begin{array}{l}\text { Grupos } \\ \text { Testes objetivos }\end{array} & \begin{array}{c}\text { Grupo I } \\ \text { No }^{\circ} \text { pac. }\end{array} & \begin{array}{c}\text { Grupo II } \\ \text { No }^{\circ} \text { pac. }\end{array} \\ \text { Schirmer } & 2 & 25 \\ \text { BUT } & 2 & 25 \\ \text { Rosa bengala II / III } & 4 & 27\end{array}$

Grupo I - pacientes HIV+ (sem diagnóstico clínico e laboratorial de AIDS); Grupo II - pacientes com AIDS (manifestação clínica e/ou contagem de TCD4+ abaixo de 200 células $/ \mathrm{mm}^{3}$ ).

No pac.: Número de pacientes; BUT: Tempo de ruptura do filme lacrimal

\begin{tabular}{|c|c|c|}
\hline $\begin{array}{r}\text { Grupos } \\
\text { Conduta terapêutica }\end{array}$ & $\begin{array}{l}\text { Grupo I } \\
\mathrm{N}^{\circ} \text { pac. }\end{array}$ & $\begin{array}{l}\text { Grupo II } \\
\text { No pac. }^{\circ} \text { pact }\end{array}$ \\
\hline Ausência de HAART & 0 & 5 \\
\hline HAART & 2 & 11 \\
\hline Total & 2 & 16 \\
\hline
\end{tabular}

Grupo I - pacientes HIV+ (sem diagnóstico clínico e laboratorial de AIDS) Grupo II - pacientes com AIDS (manifestação clínica e/ou contagem de TCD4+ abaixo de 200 células $/ \mathrm{mm}^{3}$ ).

No pac.: Número de pacientes; HAART: Terapia anti-retroviral combinada

internas após a introdução do HAART ${ }^{(22)}$; contudo, o mesmo não foi observado com relação às manifestações oculares externas. Neste estudo, a frequiência de olho seco foi superior a $20 \%$, comparável a vários estudos em pacientes com teste anti-HIV positivo, anterior ao HAART, que já relatavam a alta 
286 Estudo da síndrome da ceratoconjuntivite seca de pacientes soropositivos para o vírus da imunodeficiência adquirida humana tipo 1 e com síndrome da imunodeficiência adquirida, em uso ou não de terapia anti-retroviral combinada (HAART)

incidência de olho seco em crianças e adultos contaminados com o vírus ${ }^{(11-13)}$. Os relatos variam quanto à incidência, observando uma variação de 12 a 80,29 \% de pacientes que já apresentavam algum tipo de sofrimento celular (rosa bengala) ou que tinham teste de Schirmer anormal.

O presente estudo mostrou que a queda na produção lacrimal, mensurada pelo teste de Schirmer ocorreu em cerca de $22,5 \%$ dos pacientes. Entretanto, o decréscimo da produção lacrimal não esteve relacionado com a severidade da infecção pelo vírus da imunodeficiência adquirida $(\mathrm{p}>0,05)$.

Dos pacientes que apresentaram queixas sugestivas de ceratoconjuntivite seca, 58,06\% apresentaram algum grau de metaplasia escamosa do epitélio conjuntival, sendo que os níveis dos linfócitos T CD4+ variaram de 9 a 880 células / $\mathrm{mm}^{3}$, mostrando que não há influência da queda dos níveis destes linfócitos, normalmente observada nos pacientes contaminados, na síndrome de olho seco nos pacientes HIV positivos.

Entre os pacientes que apresentaram metaplasia escamosa, houve diferença significativa entre os pacientes que estavam em uso de HAART e aqueles que não estavam em uso de medicação.

Deve-se lembrar que, apesar da citologia de impressão conjuntival ser um teste útil para diagnóstico de olho seco, existem variações regionais na superfície conjuntival que dificultam sua interpretação, com maior tendência a apresentar aspecto normal (grau “0”).

Devido à escassez de publicações relacionando a freqüência de síndrome de olho seco após a introdução do HAART, dificulta a comparação deste estudo com outros estudos.

Outro dado importante é que, enquanto na população em geral soronegativa para o vírus HIV, conforme literatura pesquisada $^{(21)}$, há uma maior prevalência de olho seco em mulheres, neste estudo observou-se predominância no sexo masculino; isto sugere que a síndrome de olho seco nos pacientes soropositivos não está relacionada ao sexo.

Entre os pacientes com AIDS que apresentaram metaplasia escamosa do epitélio conjuntival, $68,75 \%$ faziam uso de terapia anti-retroviral oral combinada. Isto sugere que além da disfunção lacrimal, pode estar ocorrendo ceratites tóxicas ou inespecíficas, provavelmente provocada pela alta toxicidade dos diversos medicamentos sistêmicos administrados a esses pacientes. Estas alterações podem tornar os pacientes soropositivos vulneráveis a doenças infecciosas exógenas.

Devido ao importante aumento da sobrevida dos pacientes, é esperado que a ceratoconjuntivite seca torne-se cada vez mais freqüente e que se manifeste com suas formas mais graves. Neste estudo, foi observado significativo aumento da freqüência da síndrome de olho seco nos pacientes com doença acima de 4 anos $(32,2 \%)$, principalmente entre os que estavam em uso de terapia anti-retroviral oral $(80 \%)$.

Assim, apesar da introdução do HAART, o percentual de pacientes acometidos pela síndrome de olho seco permaneceu estável, devendo sempre ser pesquisada, devido ao risco aumentado que os mesmos apresentam de desenvolver a doença, a fim de que seja iniciada a terapêutica mais apropriada, para que haja melhora da qualidade de vida das pessoas com AIDS.

\section{CONCLUSÕES}

- O diagnóstico clínico sintomático de olho seco é freqüente em pacientes portadores de AIDS $(25,8 \%)$ e baseado nos dados deste estudo, sua prevalência não mostrou diferença em relação ao sexo;

- O decréscimo da produção lacrimal nos pacientes soropositivos não esteve relacionado com a severidade da infecção pelo vírus da imunodeficiência adquirida;

- Pacientes com AIDS têm maior chance de apresentarem metaplasia escamosa do epitélio conjuntival à citologia de impressão, quando comparada com os demais pacientes soropositivos, especialmente quando o tempo de doença é superior a 4 anos;

- Pacientes em uso de HAART apresentaram uma freqüência significativamente maior de metaplasia escamosa à citologia conjuntival, quando comparada aos pacientes que não usavam HAART.

\section{ABSTRACT}

Purpose: To determine the presence of dry eye and the changes in the conjunctival epithelium of patients infected with HIV-1 and with AIDS; to investigate the relation of the changes with the characteristics and with other ocular conditions of patients; to study the influence of HAART an eyes with keratoconjunctivitis sicca in patients with AIDS. Methods: Patients with laboratory diagnosis of HIV infection, were examined and divided into two groups: I. HIV+ (without clinical diagnosis and with a CD4+ count of more than 200 cells $/ \mathrm{mm}^{3}$ ); II. Patients with AIDS (CD4+ count less than 200 cells $/ \mathrm{mm}^{3}$ and/or with clinical manifestations). The following were assessed: modes of transmission of HIV; ocular manifestations; laboratory findings, tear production and conjunctival impression cytology. Results: 43 patients of group I e 77 patients of group II were studied. The internal ocular findings were decreased significantly after the use of HAART; however, this did not occur regarding external findings. $65,1 \%$ of the patients with dry eye were men. The changes of the Schirmer test and the tear film break up time were neither related to the severity of HIV disease nor to CD4+ count. All patients with changes in the conjunctival impression cytology showed $\mathrm{KCS}$, and $88,8 \%$ of the patients were of the group II. Increase in frequency of these changes was found in patients with disease time of more than 4 years. Conclusion: The decrease in tear production has not been related to the severity of HIV infection and the use of HAART did not affect the frequency of dry eye syndrome in HIV-positive patients.

Keywords: Keratoconjunctivitis sicca; HIV seropositivity; Acquired immunodeficiency syndrome; Antiviral agents; Combined modality therapy; HIV-1/drug effects 


\section{REFERÊNCIAS}

1. Bacchetti P, Moss AR. Incubation period of AIDS in San Francisco. Nature 1989;338:251-3.

2. McClure MO, Schulz, TF. Origin of HIV. BMJ 1989;298:1267-8.

3. Mendes NF. Simian viruses, organ xenotransplantation and a hypothesis about the origin of AIDS. Braz J Med Biol Res 1993;26:231-3.

4. Barre-Sinoussi F, Chermann. JC, Rey F, Nugeyre MT, Chamaret S, Gruest J, et al. Isolation of a T-lymphotropic retrovirus from a patient at risk for acquired immune deficiency syndrome (AIDS). Science 1983:220:868-71.

5. Garry RF. Potential mechanisms for the cytopathic properties of HIV.AIDS 1989;3:683-94.

6. UNAIDS. Resumen mundial de la epidemia de VIH/SIDA - diciembre de 2001 [cited 2001 Dec]. Disponible em: URL: http://www.unaids.org.

7. Update: acquired immunodeficiency syndrome - United States, 1992. MMWR Morb Mortal Wkly Rep 1993; 42:547-51,557.

8. Cha SB, Fischman O, Barros PSM, Mikoves R. Microbiota fúngica conjuntival de pacientes com síndrome da imunodeficiência adquirida (AIDS). Arq Bras Oftalmol 1990;53:80-90.

9. Nishiwaki-Dantas MC. Olho seco. Arq Bras Oftalmol 1999;62:101-5.

10. Freitas JAH, Soranz Filho JE, Soranz JF, Barbosa ML, Gonçalves VLC. Achados oftalmológicos em pacientes com Síndrome de Imunodeficiência Adquirida. Rev Bras Oftalmol 1997;56:837-41.

11. Kadhem M; Kalisch SB, Goldsmith J, Fetkenhour C, O'Grady RB, Phair JP, Chrobak M. Ophthalmologic findings in acquired immunodeficiency syndrome (AIDS). Arch Ophthalmol 1984;102:201-6.

12. Nakanami CR, Muccioli C, Paiva ER, Abreu MT. Alterações oculares em crianças expostas ao vírus da imunodeficiência humana [resumo]. Arq Bras Oftalmol 1997;60(4)

13. Raskin RH, Justo DM, Torres LM, Picetti E, Cattani S, Bocaccio FJL, Rymer S. Avaliação de olho seco em pacientes com teste anti-HIV positivo [resumo]. Arq Bras Oftalmol 1993;56(4).

14. Steck AD, Abreu MT, Muccioli C, Lottenberg C, Junior RB. Prevalência de olho seco em população de pacientes HIV positivos [resumo]. Arq Bras Oftalmol 1994;57(4).

15. Tseng SC. Topical tretinoin treatment for dry-eye disorders. Ophthalmol Clin. 1987;27:47-53.

16. Gibard JP. Dry eye disorders. In: Albert DM, Jakobiec FA, editors. Principles and practice of ophthalmology: clinical practice. Philadelphia: WB Saunders; 2000. p.982-1000.

17. Holly FJ. Diagnostic methods and treatment modalities of dry eye conditions. Int Ophthalmol. 1993;17:113-25.

18. De Rojas MV, Rodriguez MT, Ces Blanco JA, Salori MS. Impression cytology in patients with keratoconjunctivitis sicca. Cytopathology 1993;4: 347-55.

19. Nelson JD. Impression cytology. Cornea 1988;7:71-81.

20. Rivas L, Oroza MA, Perez-Esteban A, Murube-del-Castilho J. Morphological changes in ocular surface in dry eyes and other disorders by impression cytology. Graefes Arch Clin Exp Ophthalmol 1992;230:329-34.

21. Mendes LE, Santos PM, Parente FSP, Gonçalves JOR, Belfort Júnior R. Olho seco em pessoas com idade superior a 40 anos selecionadas em 3 cidades brasileiras. Arq Bras Oftalmol 1995;58:10-5.

22. Vittinghoff E, Scheer S, O’Malley P, Colfax G, Holmberg SD, Buchbinder SP. Combination anti-retroviral therapy and recent declines in AIDS incidence and mortality. J Infect Dis 1999;179:717-20.

\section{Simpósio Internacional da Santa Casa de São Paulo}

\section{3 a 5 do Junho do 2004 \\ Centro de Conveng̣ōes Frei Caneca - SP}

INFORMAÇÕES: JDE Comunicação e Eventos

Tels.: (1 1) 287-9378 / 289-4301

Fax: (11) 288-8157

E-mail: jdecomev@uol.com.br 